\title{
ENTRE EL INDIVIDUO Y EL ESTADO: BUROCRACIA PRE-Y POST-MIGRATORIA
}

\author{
The Individual versus the State: \\ Pre- and Post-Migration Bureaucracy
}

Victoria Finn*

\begin{abstract}
Resumen. Mientras algunos estudios migratorios proponen un rol estatal preponderante, otros declaran que la globalización y el transnacionalismo tienen un poder estatal reducido. Existe una ambigüedad acerca de cuán influyente es el Estado de destino, particularmente en relación a los individuos, en materia de decisiones migratorias. En este artículo, se plantea una aproximación teórica-conceptual para analizar la relación entre el migrante (emigrante e inmigrante) como unidad individual y el Estado en regímenes democráticos contemporáneos en términos de control migratorio. Desde una perspectiva de la economía política, se clarifica cuáles son las condiciones políticas y legales necesarias y suficientes durante la trayectoria migratoria. Las decisiones estatales circunscriben quién migra e indican cuáles países de destino continúan intentando ejercer el control mediante lo que se conceptualiza en este análisis como "burocracia post-migratoria". El examinar cómo estas barreras estatales afectan la agencia de los individuos permite luego investigar la integración de los inmigrantes.
\end{abstract}

Palabras clave: burocracia post-migratoria; Estado migratorio; integración de los inmigrantes; control de fronteras; legalidad de migración.

\begin{abstract}
Some migration literature present a predominant state role, whilst others convey that globalization and transnationalism have resulted in reduced state power. There is ambiguity regarding the destination state's influence, as compared to individuals, in migration decisions. To fill this gap, I suggest a conceptual theoretical approach for analyzing the relationship between a migrant (emigrant and immigrant) and the state within contemporary democratic regimes of migratory control. Through a political economy lens, I define which political and legal aspects are necessary and sufficient conditions during the migrant trajectory. State decisions dictate who migrates and show which destination countries continue to attempt to control migration, through what I define as 'post-migration
\end{abstract}

PhD Candidate Universidad Diego Portales, Santiago, Chile; Leiden University, Leiden, The Netherlands. Santiago, Chile. E-mail: vickijfinn@gmail.com. Orcid: 0000-0001-6379-3720. 
bureaucracy.' Examining how state limits affect individuals' agency allows for supplementary research on immigrants' integration.

Keywords: post-migration bureaucracy; migration state; immigrant integration; border control; illegal migration.

\section{Introducción}

Existe un proceso de toma de decisiones y formulación de políticas públicas que condicionan quién está presente "legalmente" en un país de destino y, por tanto, quién tiene la posibilidad de participar formalmente en la sociedad. Aunque la decisión de (no) mudarse se origina en los individuos racionales, sus opciones están siempre limitadas y definidas por leyes migratorias. Las barreras estatales no siempre son eficientes en controlar la migración dada la autonomía de los propios migrantes (los emigrantes y los inmigrantes) (Mezzadra, 2015); no obstante, una persona racional considera toda la información disponible, incluso las reglas estatales del país de destino. Desde esta aserción se reconoce el rol importante e innegable del Estado como actor protagónico en el fenómeno de la migración internacional. Este artículo propone una aproximación teóricaconceptual de los límites políticos con el objetivo de estudiar, en una manera sistemática, la relación entre la toma de decisiones de los individuos con la burocracia y las barreras estatales involucradas en el fenómeno migratorio.

La literatura acerca de la pre-migración (sobre un individuo antes de migrar) ofrece extensas razones para la ocurrencia de la migración, utilizando diferentes explicaciones como la teoría del sistema mundial, los factores básicos de empuje y atracción, y las estrategias a nivel del hogar para diversificar las fuentes del ingreso familiar (Massey et alii, 1993). Todos estos motivos se traducen en decisiones racionales tomadas por los individuos y las familias en los circuitos migratorios (Rivera Sánchez, 2012). Además de conocer por qué algunos individuos optan por migrar mientras que otros no, existe una vasta literatura sobre la era de control migratorio con respecto a los regímenes globales de migración, el control de fronteras, la legalidad de migración y la criminalización de la movilidad (Bigo, 2004; Bigo, 2015; Mármora, 2010; Domenech, 2013; Domenech, 2015; Estupiñán Serrano, 2013; Hollifield et alii, 2014; De Genova et alii, 2015; Domenech, Pereira, 2017).

A diferencia de las investigaciones mencionadas, el objetivo de este artículo es analizar cómo y qué barreras definen las condiciones para establecer quiénes se pueden migrar. Este artículo también se interesa en quiénes son los que definen dichas condiciones. La aproximación teórica-conceptual se surgió orgánicamente del conjunto de la amplia literatura multidisciplinaria previamente mencionada. Se considera a las restricciones pre-migratorias como todas aquellas que se originan desde el Estado. Bajo este contexto, existen varios actores involucrados, tanto desde el país de origen como desde el país de 
destino. El colectivo del Estado está compuesto de individuos, con la diferencia de que contiene representantes del electorado y trabajadores estatales. En base a estos actores y sus perspectivas, surgen preguntas de migración que se pretenden responder en este artículo; ¿cómo y cuándo se forma la relación migratoria del individuo con el Estado? Más aún, ¿̇es la agencia del individuo o el Estado el elemento más poderoso en dicha relación? Y posteriormente, ¿cómo afectan las decisiones del país de destino al proceso de toma de decisiones de los individuos que desean migrar?

Revisando este proceso, se debe enfatizar el conjunto de individuos que componen el grupo de posibles migrantes. Después, una vez definido este grupo reducido, se debe derivar del mismo grupo, un subconjunto de inmigrantes en un país de destino. Este artículo asume una perspectiva de la economía política, que considera a los individuos como unidades racionales - pero falibles y con información limitada - con la posibilidad de cambiar las instituciones que los condicionan. La racionalidad en sí misma no explica el motivo de mudarse, sino más bien la decisión de migrar es un acto racional. Entendida en esta manera, la racionalidad permite aplicarse a un fenómeno tan complejo como las trayectorias de migración heterogéneas. A su vez, en este artículo se incluye argumentos para entender no solo los límites considerados por un individuo antes de migrar, sino también la composición y el origen de los límites políticos del fenómeno migratorio. Se propone entonces una aproximación teórica-conceptual que permita manejar dichos límites políticos que estudie a priori quién migra.

Dado que la última etapa de la propuesta conceptual es la participación, esta aproximación sirve como punto de partida para los estudios de integración. De ahí que en este artículo se utilizan las ideas de "integración" y "participación" en lugar del término anacrónico "asimilación", ya que la integración completa es equivalente al acceso a la participación completa. La integración no es lineal ni igual para todos. En efecto, esta reflexión ayuda a acotar el universo de aquellos que se podrían considerar a sí mismos como posibles migrantes y entender las etapas que tuvieron que pasar aquellos que son finalmente el subconjunto de inmigrantes en un país de destino.

La siguiente sección presenta la base teórica relevante para estudiar la relación entre los individuos y los Estados con respecto a la migración y la participación. En el segundo apartado se plantea y se explica una nueva aproximación teórica-conceptual, basada en la interpretación de conceptos y teorías migratorias, agregando temporalmente la burocracia pre- y post-migratoria. Esta contribución permite luego estudiar los nexos post-migratorios - por ejemplo, integración versus segmentación, participación versus no-participación, inclusión versus exclusión. En la tercera sección, el 
foco del Estado se estructura en tres: 1. el rol del Estado migratorio, 2. el control de fronteras y 3 . la legalidad de migración.

\section{El rol del migrante en un mundo globalizado}

En este artículo, se asume que todos los individuos son racionales, ya que estos estiman los beneficios y costos (no necesariamente monetarios) de migrar. La decisión migratoria es particular en cada caso porque la utilidad (el beneficio) subjetiva de migrar varía y depende de las preferencias del individuo. Por ejemplo, algunas personas valoran más la seguridad, otras el empleo o la libertad de religión. Las preferencias y valores deseados están reflejados en las prioridades y acciones de los individuos. A pesar de que no logramos nunca entender la combinación exacta de los motivos específicos en la decisión final de cada migrante, se puede asumir que el individuo tomó la decisión basada en sus propios intereses (que no son necesariamente egocéntricos), para mejorar algún aspecto de su calidad de vida personal o familiar ${ }^{1}$.

Considerando la complejidad de nuestro mundo social, la información perfecta y completa es inexistente, en consecuencia se puede observar una forma de racionalidad limitada (Ostrom, 2011). Es decir, las personas poseen información incompleta; sin embargo, buscan tomar la mejor decisión posible en su contexto. Esto también es aplicable a la hora de tomar decisiones migratorias porque las reglas y las políticas estatales limitan el ejercicio de la agencia por parte de los individuos. Una persona racional considera toda la información disponible, incluso las reglas estatales del país de destino. Se reconoce entonces el rol protagónico del Estado en la migración internacional desde el principio.

Las estructuras multiniveles de poder político y económico pueden impactar en el discurso y estructuras de poder a nivel global, capturado en la generación de un "nuevo" paradigma de movilidad (Kalm, 2008, p. 135). La globalización de la migración conlleva numerosas influencias teóricas y prácticas externas más allá de la región, sobre todo en términos de los regímenes globales, complementados con su propio vocabulario, problematización y objetivos. Por ejemplo, el concepto de "la migración ordenada" que es utilizado a nivel global para poder gobernar el fenómeno de la migración, es una idea promovida por la Organización Internacional de Migración. Otros elementos globales que generan el vocabulario y definen la ideología y los conceptos migratorios son los diálogos y foros globales sobre migración, con el resultado de "la construcción y consolidación de una serie de consensos acerca de los modos adecuados de administrar, gestionar o gobernar la migración a escala global" (Domenech,

Bajo esta perspectiva de racionalidad, también se aplica la migración por motivos humanitarios. Mudarse a otro país, mejora la calidad de vida del individuo que requiere atención humanitaria. 
2013, p. 5). Existen también intentos de consensos de políticas públicas para gobernar el fenómeno migratorio a nivel regional (véase por ejemplo, Finn et alii, 2019).

La re-estructuración de la migración a causa de la globalización origina nuevas dinámicas entre los mercados internacionales, los Estados y los individuos. Esta re-estructuración evidencia un contexto en específico, imponiendo desafíos, mas no la eliminación de los Estados (Bigo, 2004, p. 3). Son las relaciones internacionales y las tensiones entre países que determinan el ámbito político en el mundo globalizado, generando la posibilidad de ejercer en el caso de los individuos, una lealtad (ciudadanía) nacional única o múltiple (Waldinger, Fitzgerald, 2004, p. 1178). No obstante, un ambiente político que tolera, y además apoya, el nacionalismo del extranjero en el país de destino, o permite ejercer múltiples grados de lealtad de los migrantes, no debería darse por hecho ni como una norma (Waldinger, Fitzgerald, 2004, p. 1188). La situación actual de lealtad múltiple no habría existido como fenómeno en periodos anteriores a la era de la globalización. Habría sido imposible concebirlo antes del fin de la Guerra Fría, porque un contexto de guerra destruye la posibilidad de una lealtad dual e imposibilita el nexo transnacional (Faist, 2000, p. 219). Dados los efectos de la globalización, una ciudadanía nueva es inevitable (Bauböck, 1994). El territorio existe como elemento clave en el mundo político y las relaciones migrante-Estados son por ende insoslayables. Como tal, “(...) la migración es tanto una causa como una consecuencia de los cambios políticos y económicos. La migración internacional, parecida al comercio, es una característica fundamental del orden liberal posguerra" (Hollifield, 2004, p. 905). Estas relaciones estatales internacionales existen conceptualmente al borde de la Figura 1 (en Sección II) en la interacción entre países de origen y de destino.

En países democráticos, el Estado es una composición de individuos que son representantes estatales (por ejemplo, trabajadores públicos o funcionarios en puestos elegidos). En otras palabras, los individuos componen lo que se relaciona con el poder estatal democrático. Esta relación se explica porque los gobernantes son los agentes elegidos por los votantes que actúan como principales (Powell, 2004, p. 274). Sin embargo, los derechos políticos no garantizan que las políticas públicas y las reglas sean siempre fieles a las necesidades de los votantes. Además, existe una diferencia entre los objetivos (planeados) de las políticas públicas de inmigración y los resultados de dichas políticas, denominada la hipótesis de brecha (gap hypothesis) (Hollifield, 1986; Hollifield et alii, 2014; Hollifield, Wong, 2015).

Varios estudios migratorios usan al Estado como una unidad o un nivel de análisis (Hollifield, Wong, 2015, p. 240). Pero otros, como por ejemplo la contribución de Glick Schiller (2010a, p. 110), critica el uso del Estado como 
una unidad de análisis; el considerar un país y sus fronteras declaradas podría ser interpretado como nacionalismo metodológico (Wimmer, Glick Schiller, 2002; Glick Schiller, 2010a)². Una alternativa es trabajar empíricamente con un nivel meso o micro, como es el caso de este artículo. No se considera el Estadonación en manera normativa, tampoco como un "contenedor", ni como la unidad natural para entender fenómenos sociales. En cambio, se percibe que un grupo de individuos, como votantes en una sociedad democrática, determinan las condiciones políticas y legales implementadas por representantes estatales.

El enfoque en el individuo como unidad de análisis evita un vocabulario unidimensional ("nativo" o "migrante"), que conlleva a dos beneficios. Uno, los residentes en los países democráticos continúan recibiendo más derechos políticos en el destino, lo que significa que existe una extensión de la posibilidad de participar políticamente, aún sin poseer la ciudadanía, y que se traduce luego en tener una voz para imponer demandas al Estado. Esta extensión de derechos a residentes no-ciudadanos (i.e. inmigrantes) sucede en paralelo con una extensión de derechos de los ciudadanos en el extranjero (i.e. emigrantes) (Arrighi, Bauböck, 2017). Dos, el grupo de residentes es heterogéneo, lo que significa que poseen preferencias políticas distintas entre ellos. Los individuos simultáneamente están en varios grupos (por nacionalidad, etnicidad, raza, género, extranjero/a) y la combinación - o la interseccionalidad - de dichos grupos tiene un efecto en la posición de poder del individuo (Viveros, 2016). Como se plantea en este artículo, algunos de estos grupos de clasificación en el proceso migratorio están formados por y desde el Estado. Estas clasificaciones afectan directamente a los grupos puesto que determinan quiénes están presentes como inmigrantes en un país de destino, y después, la oportunidad de cada individuo de ejercer un grado de participación en la sociedad, así como de relacionarse con las instituciones formales del país de destino.

\section{La participación}

La integración depende no solo de los inmigrantes - si la desean - sino también de la sociedad "dominante" que impone límites a dicho proceso (Berry, 1997, p. 27)3. La inclusión y exclusión en la sociedad no son dicotomías fijas, sino que existe un continuum entre los dos términos (por ejemplo, niveles distintos de estratificación social). El contexto sociopolítico define la toma de

2 El nacionalismo metodológico es el supuesto de que la nación/Estado/sociedad es la forma natural, lógica, política y social para entender los fenómenos del mundo moderno (Wimmer, Glick Schiller, 2002, p. 302). Glick Schiller (2010a, p. 110-111) ha actualizado dicha definición como una orientación ideológica que estudia a los procesos sociales o históricos como si ocurriesen y estuviesen contenidos dentro de las fronteras estatales, en cambio de dentro de los sistemas globales.

3 Berry (1997) considera cuatro posibilidades post-migratorias: integración, asimilación, separación o segregación, y marginalización. 
decisiones estatales y también clarifica el proceso de interacción formal entre las políticas públicas y los individuos.

Dichas interacciones pueden resultar en distintos niveles de participación. No se considera la participación total como un objetivo primordial del individuo ni del país de destino, dado que no todos los inmigrantes desean integrarse en un sentido completo; y en paralelo, no todos los Estados desean que los inmigrantes se integren de forma completa. Dado que existen varios niveles de participación formal, cabe preguntarse: ¿qué o quién decide el resultado de dicho proceso y para quién? Para poder responder a esta pregunta, se plantea que los estratos del proceso de migrar, bajo las restricciones estatales pre- y post-migratorias, juegan un rol clave en definir qué tipo específico de integración está disponible para cada individuo.

Esta nueva aproximación que se propone - en base a las interacciones entre la agencia del individuo y el Estado - se contrapone a aquellas visiones del fenómeno migratorio que solo consideran a cada migrante como un simple agente auto-seleccionado. Conceptualmente, la auto-selección omite una parte del fenómeno sociopolítico que compone las relaciones en varios niveles entre los migrantes y el país de destino. Se incluye la pre-relación entre el individuo y el Estado, que a su vez circunscriben los subsiguientes procesos post-migratorios de participación.

\section{La aproximación teórica-conceptual}

El saber que un individuo racional migra para mejorar su condición personal, o la de su familia - al ejercer su agencia - no es un motivo suficiente para después explicar un fenómeno tan complejo como la participación social o política en un país. El proceso de auto-selección y ejercicio de la agencia está limitado por barreras pre-establecidas en varios niveles por el país de destino. Esta forma de entender cómo la agencia y la racionalidad del individuo al momento de migrar, está acotada por las condiciones estatales. Bajo este contexto, se reconcilia conceptualmente la idea de agencia en la migración y el concepto de Estado como fuente de acción. El incluir la relación del migrante-Estado tanto en la pre- como en la post-migración, permite iluminar el proceso de participación formal de los inmigrantes en el país de destino.

Los límites políticos y legales definidos por los territorios soberanos forman una relación simbiótica entre potenciales migrantes y Estados, que dan forma a una relación pre-migratoria del individuo-Estado. Una vez que se cruzan las fronteras, los inmigrantes que cumplen la burocracia post-migratoria, en comparación a aquellos que no la cumplen, poseen una relación distinta con el Estado. No obstante, todos aquellos que cruzan la frontera forman la población de inmigrantes que enfrentan la posibilidad - siempre distinta - de participar. 
El resultado final es una población acotada de inmigrantes, que se visualizan gráficamente en la Figura 1.

Figura 1 - Los estratos de la relación migrante-Estado en el proceso migratorio hacia la participación

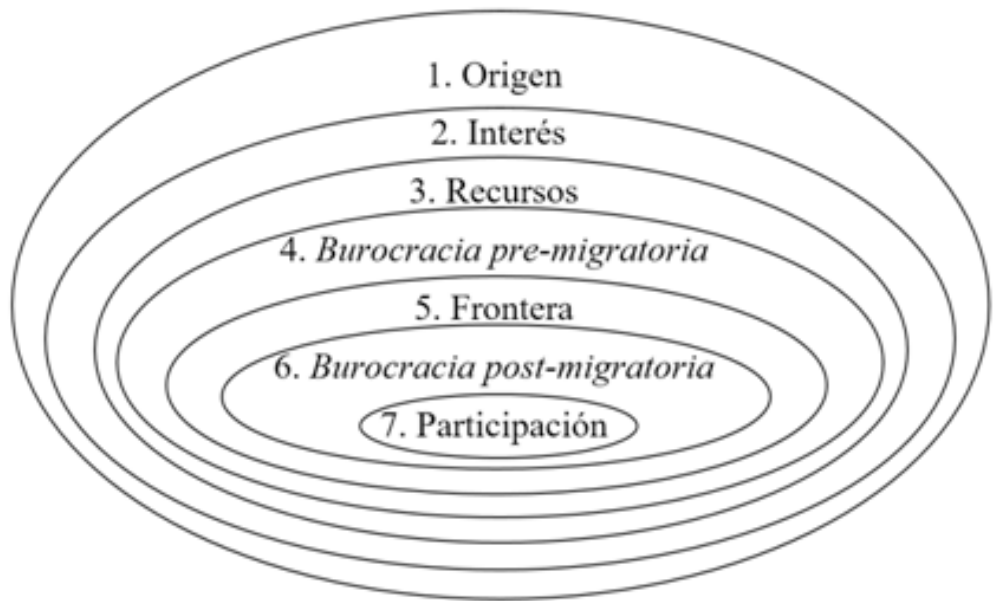

Fuente: Elaboración propia basada en teorías de migración y el Estado migratorio (e.g. Massey et alii, 1993; Hollifield, 2004).

Notas: El gráfico muestra la conformación de los grupos durante trayectorias migratorias heterogéneas. Los conjuntos no grafican una proporción a escala. Los estratos en cursiva pueden ser pasados o exonerados, pero con repercusiones.

La Figura 1 involucra individuos de un país de origen y de un país de destino. Esta aproximación teórica-conceptual muestra la reducción de la posible población extranjera en un país de destino a través del siguiente proceso político:

1. individuos en la población de los países de origen;

2. grupos o individuos con un interés plausible en migrar;

3. grupos o individuos con suficientes recursos para considerar migrar;

4. individuos que logran superar la burocracia pre-migratoria (por ejemplo, las visas);

5. individuos que pasan exitosamente por la frontera;

6. inmigrantes que logran superar la burocracia post-migratoria del país de destino;

7. inmigrantes que confrontan la posibilidad de participar en la sociedad de destino.

La agencia de los individuos y el Estado están presentes e interactúan en toda la trayectoria migratoria, en vez de necesariamente competir entre ellos. 
En la aproximación, se argumenta que los límites y condiciones - impuestos a través de leyes, burocracia y control de fronteras - establecidos por un país de destino, no necesariamente alteran las preferencias de los individuos de forma endógena, sino que ponen las condiciones ex ante. Estas "reglas del juego" influencian la trayectoria migratoria. Como resultado, las restricciones disminuyen el grupo de individuos que migra.

El estrato 1 representa a todas las personas en otros países que constituyen un universo potencial de migrantes, pese a que no toda esa población desea mudarse a nivel internacional. La auto-selección se visualiza ya en el estrato 2 conformado por las personas con un interés en migrar. El estrato 3 se compone de aquellos que poseen o pueden obtener los recursos (económicos y temporales) necesarios para superar las barreras burocráticas del proceso para cumplir con el interés de mudarse al extranjero.

Los migrantes son únicos no solo en comparación con los individuos de otros países (si se mide sus diferencias a través de la brecha de asimilación entre estos grupos), sino que también son diferentes al resto de la población en el origen. Estos tienen distintos recursos y son auto-seleccionados para mudarse al extranjero (es decir, por ejercer su agencia a la hora de migrar). Después de la migración, los no-migrantes en el país de origen (left-behind) también pueden cambiar sus características, por las vinculaciones transnacionales entre los migrantes y no-migrantes; por ejemplo, la existencia de la separación geográfica, pero al mismo tiempo la existencia de responsabilidades de remesas, son las que establecen relaciones transnacionales de poder en sistemas globalizados (Glick Schiller, 2010b, p. 38-41). En el destino, existen diferencias entre los nuevos llegados y aquellos ya establecidos con respecto al nivel de membresía en la democracia (Bauböck, 2012).

Este artículo se concentra particularmente en los componentes relacionados a los estratos 4,5 y 6 que son las directrices establecidas por el Estado, por lo que representan el poder o el control estatal del fenómeno migratorio. En otras palabras, los estratos 4, 5 y 6 evidencian el rol del Estado como actor de migración internacional desde la relación individuo-Estado. Estos números no implican una tendencia lineal, ya que un individuo podría revertir o repetir los pasos de cada estrato. Las decisiones tomadas en los estratos 4, 5 y 6 reflejan la autonomía de los migrantes de cómo "aceptan, rechazan, desobedecen, negocian y resignifican las políticas de control migratorio" (Alvites Baiadera, 2018, p. 176).

Es específicamente en el estrato 4 de la burocracia pre-migratoria (por ejemplo, la visa de entrada) cuando comienza la relación entre el individuo como migrante y el país de destino. Los sistemas de pasaportes y visas - en papel o digital - y las instituciones de nacionalidad y de ciudadanía, 
que se establecieron en los siglos XIX y XX, son componentes integrales de lo que reconocemos como el Estado-nación moderno (Hollifield, Wong, 2015, p. 232). Las instituciones típicas y simbólicas del Estado-nación y las diferenciaciones entre países a través de conceptos como identidad nacional, símbolos como banderas y el uso de pasaportes han sido fomentadas desde las fronteras y las barreras de entrada. Entonces, al aumentar la noción de pertenencia a un territorio de contrapartida también aumenta la exclusión de una persona "no-nacional", impidiendo a la misma el poder entrar y vivir en dicho territorio.

La frontera, el estrato 5 en la Figura 1, se transforma en un muro invisible que otorga solo a algunas personas el poder pasar a otro Estado-nación. También la frontera sirve para negar la entrada de otros individuos, entonces está utilizada como una herramienta política de exclusión legitimada por el Estado (De Genova et alii, 2015, p. 3). El cruce de frontera es un momento de incertidumbre que los inmigrantes exitosos han "sobrevivido" (Alvites Baiadera, 2018, p. 183). El control o la seguridad de la frontera es crítico para la soberanía; según Hollifield y Wong (2015, p. 229, 236), el "control" de migración se refiere exactamente al rol estatal que establece reglas de entrada y salida, con la idea de que la entrada es más problemática, pues es más controlada que la salida. Se debe señalar entonces que la introducción de la burocracia estatal - la invención de nuevas realidades fronterizas operacionaliza la migración, para después poder gobernarla y limitarla a través de un régimen de gobernabilidad estatal con prácticas políticas que desciendan desde el Estado-nación, que a su vez legitima y fomenta dichas realidades (Kalm, 2008; Geiger, Pécoud, 2010; De Genova et alii, 2015, p. 13).

Los términos 4 y 6 de dichos estratos están en cursiva porque es posible, en la práctica, saltar estos dos pasos en el camino migratorio. Esta situación surge cuando un individuo logra cruzar la frontera sin cumplir con la burocracia pre-migratoria y cuando reside en aquel país de destino sin cumplir con la burocracia post-migratoria. No obstante, dicha situación cambiaría completamente la relación del individuo-Estado. Sin cumplir los requisitos de la burocracia pre- y post-migración, el inmigrante se queda sin todos los documentos necesarios, pues no sería considerado como "legal" en el país de destino. La falta de documentos aumenta la paranoia de los votantes, visto en la construcción de la criminalización de "migrantes ilegales" (Bigo, 2015).

Los individuos considerados "irregulares", "no documentados" o "ilegales" en el país de destino también pueden estar en el estrato 7, pero poseen un estatus que primero afectará sus interacciones con la sociedad y las instituciones formales, y segundo incidirá en su participación. Investigadores aseveran que una persona "ilegal" es más que excluida, dado que el término se fracciona en un amplio vocabulario de matices de regularización, que a su 
vez afecta su recepción en la sociedad de destino (Mezzadra, 2015, p. 16). La decisión de evadir estos estratos implica resultados previamente conocidos por el inmigrante. Este individuo tiene conocimiento de que esto afectará su posición, poder y posibilidad de participar en el país de destino, reduciéndola de forma considerable. Sin todos los documentos formales, típicamente se reduce su esfera de interacción con el mercado formal del trabajo y con la sociedad, debido a la dificultad o la imposibilidad de obtener un contrato, acceder al sistema bancario, o inscribirse en la educación superior. Así mismo, se complica el acceso de este inmigrante al uso de sistemas nacionales (salud o educación pública). En definitiva, a pesar de lograr entrar al estrato 7 del proceso, si alguien omite algunos pasos previos, en particular el 4 y 6 de la burocracia, su posición en términos de oportunidades de integración a través de la participación formal, se reduce drásticamente. Estos pasos claves así como los posibles resultados negativos en caso de obviarlos, demuestran la relevancia del rol del Estado en los procesos de migración y en los subsiguientes procesos de integración.

Hollifield (2004, p. 885) argumenta que las condiciones suficientes para la ocurrencia de fenómenos migratorios son las legales y las políticas. $\mathrm{Al}$ enfocarse en las condiciones estructurales, Hollifield subestima el poder - o agencia - del individuo, obviando implícitamente las relaciones claves del individuo-Estado. Por ende, se observa que las interacciones durante el proceso migratorio suceden entre individuos, pero que las políticas y burocracias estatales construyen el contexto que las condicionan y constriñen. Esta observación nos indica que no se puede desestimar la relación formal pre- y post-migratoria entre el individuo y el Estado. Los estratos de la Figura 1 demuestran que los migrantes no simplemente aceptan las condiciones estructurales de un país de destino, sino que son agentes involucrados en el proceso. Mientras las democracias ofrecen más derechos políticos tanto a los ciudadanos como a los no-ciudadanos, "la voz de la sociedad" es siempre más heterogénea, e incluso más transnacional debido a los flujos entre los países de origen y destino. El resultado de los primeros seis estratos ayuda a visualizar la reducción de la composición del grupo efectivo en el último estrato 7, que sirve posteriormente como un punto de partida en los estudios de participación de los inmigrantes.

El proceso político de la relación migratoria del individuo-Estado, reflejado en toda la Figura 1, puede crear posteriormente los derechos estratificados de movilidad (Kalm, 2008, p. 135). En otras palabras, resultan en estratificaciones políticas y legales a nivel individual, influenciadas por el Estado antes de la llegada; que luego pueden ser exacerbadas en la post-migración. Es en este preciso momento en donde se hacen evidentes las implicancias conceptuales y prácticas que llevan la idea de la relación formal entre la agencia del 
individuo y el Estado. Dicha relación logra reflejar inherentemente procesos universales, sin la necesidad de tomar explícitamente una "perspectiva global" o transnacional, que parece reducir el rol del Estado (Glick Schiller, 2010a). Aunque las relaciones transnacionales pueden disminuir los costos de migrar, el Estado todavía controla las políticas públicas migratorias que afectan el proceso de la toma de decisión de migrar.

\section{El Estado migratorio democrático: el control y la legalidad}

En esta sección, se busca posicionar el rol estatal como poder político y como ejercicio de soberanía, en tanto es uno de los actores principales que sedimenta el contexto institucional del fenómeno de migración. El Estado no es un actor en sí mismo, ya que está compuesto de individuos e influenciado por muchas fuentes, por ejemplo organizaciones y prácticas internacionales (Geiger, Pécoud, 2010). El contexto internacional actual está centrado en un sistema político de múltiples y dispares Estados-nación, cuyas fronteras no están desterritorializadas por las comunidades ni individuos transnacionales, sino que se estructuran mediante el uso de fronteras territorializadas, administradas y definidas por los mismos Estados-nación.

En las democracias, la demanda de políticas públicas de inmigración depende de los intereses organizados y la distribución de los costos y beneficios (Freeman, 1995). En un régimen democrático transparente, el cómo está definida la seguridad nacional - por ejemplo el uso de las visas y el control de la frontera - depende indirectamente de los votantes que eligen a sus gobiernos. Vale decir, “(...) en una relación representativa, se puede concebir a los ciudadanos como principales representados por agentes que los ciudadanos mismos han delegado temporalmente el poder de crear las políticas públicas" (Powell, 2004, p. 274). Las demandas de la sociedad entonces influyen en la toma de decisiones de un gobierno. Más aún, el contexto legal - por ejemplo, en los sistemas democráticos definidos por una constitución - limita la posibilidad de crear políticas arbitrarias.

Existen posiciones discordantes dentro de democracias que juegan un rol en una política desarticulada: a nivel estatal, algunas leyes son a menudo contradictorias, especialmente en Estados liberales (Hampshire, 2013). Se puede encontrar que las políticas liberales resultan en beneficios económicos, pero que son inherentemente yuxtapuestas a las presiones políticas que demandan más restricciones del fenómeno a nivel local. Esta tensión es inherente en sistemas liberales de representación política moderna a lo que Hollifield (2004, p. 885) ha denominado como la "paradoja liberal". Dado que los residentes en democracias más liberales tienen más derechos de auto-expresión, sus voces acarrean más poder al determinar políticas públicas. Según Hollifield y Wong (2015, p. 236), es precisamente en dichas democracias que es más 
probable que el control de migración se convierta en un tema político y por ende, contradictorio. La razón de la coexistencia es simple: los actores internos (stakeholders) poseen una variedad amplia y heterogénea de objetivos y visiones del mundo, los mismos que se traducen en demandas variopintas acerca de la migración para con el gobierno (Hampshire et alii, 2014).

El contexto actual de un país también afecta las posturas de los residentes del país de destino hacia los nuevos grupos residentes. La Tabla 1 resume lo planteado hasta ahora.

Tabla 1 - Las relaciones y condiciones pre- y post-migratorias

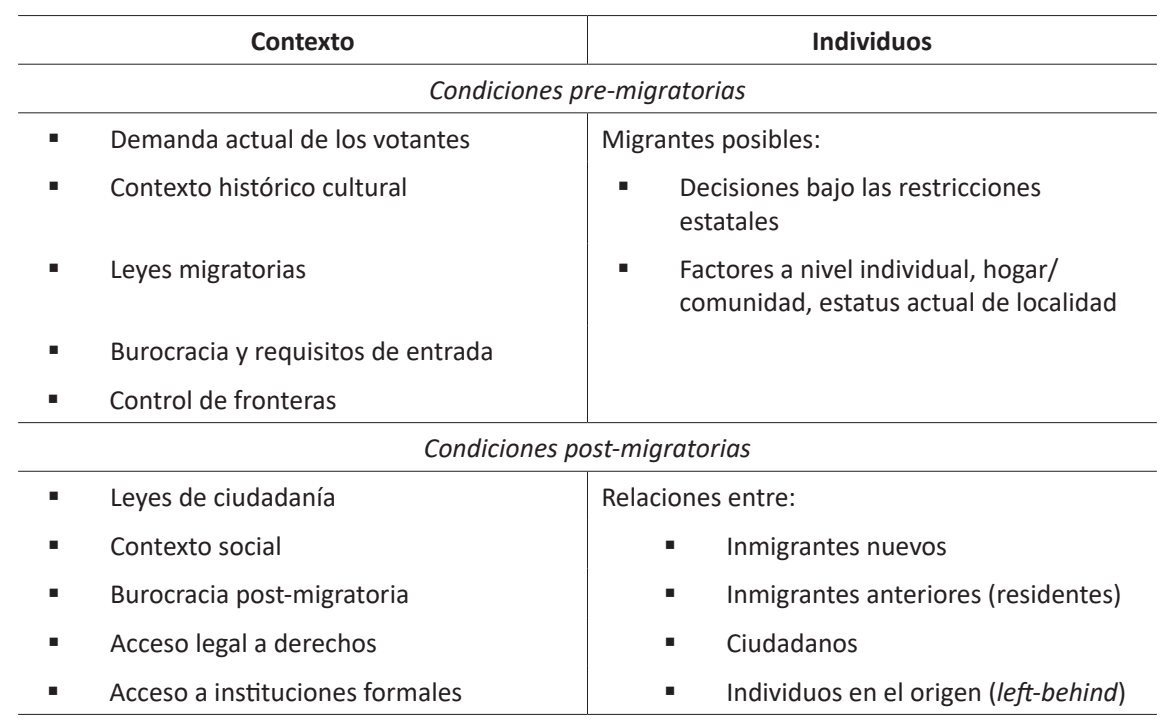

Fuente: Elaboración propia.

Notas: Las demandas de la población están basadas en los resultados de las políticas públicas migratorias (Hollifield, 1986), que a su vez determinan las leyes, el nivel de burocracia y la seguridad de la frontera.

\section{El control de fronteras}

En términos definidos por Hollifield (2004), existe el "Estado migratorio" que trata de gestionar la migración durante la expansión de la globalización. En sí mismo, la migración no es un fenómeno que requiere necesariamente una gobernanza explícita. No obstante, la época actual de control político del Estado tiene sus orígenes en un proceso histórico de regímenes globales de migración, control de fronteras, legalidad de migración, criminalización de la movilidad solo para con algunas personas y vigilancia de los extranjeros post-migración (Bigo, 2004; Bigo, 2015; Stumpf, 2006; Kalm, 2008; Mármora, 2010; Estupiñán Serrano, 2013; Hollifield et alii, 2014; De Genova et alii, 2015; Domenech, 2015; Domenech, Pereira, 2017). 
Los Estados deciden entre dejar que la migración opere libremente en el mercado (a través del intercambio de los individuos que ejercen su agencia) o tomar un rol activo en su gestión (Kalm, 2008, p. 169). Con el objetivo de mantener su soberanía y legitimidad, los Estados generalmente eligen un modelo de gobernabilidad migratoria (Mármora, 2010, p. 71). No obstante las deficiencias, los modelos de gobernabilidad migratoria continúan intentando mantener la legitimidad estatal en una época de globalización en donde el concepto de Estado-nación está siendo fuertemente cuestionado. Los resultados de la decisión de ejercer control se visualizan en los estratos 4,5 y 6 de la Figura 1, a través de la relación del Estado con los individuos en su trayectoria migratoria. No obstante, existe un grupo de individuos que pasa exitosamente por la frontera (el estrato 5), después de haber superado el estrato 4 de la burocracia pre-migratoria como la única ruta legal de entrada. Entonces, cumplir con los requisitos estatales implica poseer los documentos necesarios para residir en el país de destino. Este proceso burocrático crea una situación en la cual, sin los documentos, una persona es considerada como irregular y por tanto, no-autorizada, lo que a su vez crea la idea de una persona "ilegal" en el territorio.

La reacción estatal de decidir gestionar la migración puede ser interpretada como un intento para poder mantener - o aparentar mantener la soberanía nacional; esto con el propósito de sostener su relevancia políticainternacional como Estados-nación, frente a la re-estructuración causada por la globalización. En la era actual del control migratorio, la existencia de personas sin documentos en localidades representa la pérdida de control de las fronteras por parte del Estado; De Genova (2002, p. 436) describe este fenómeno como el "espectáculo de la frontera". Para evitar esta imagen, un Estado puede tratar de "pre-seleccionar" a los individuos mediante la facilidad o dificultad de entrar al país, reflejados en los estratos 4 y 5 . Dichas políticas selectivas son un intento de control para poder influenciar ex ante en la decisión individual de migrar al exterior. Estos individuos se componen los estratos 2 y 3.

Un país de destino entonces define cuáles flujos migratorios son deseables e indeseables basado en "(...) su carácter ordenado/desordenado, voluntario/forzoso y reducido/masivo" (Domenech, 2013, p. 6). Los requisitos de entrada demuestran la deseabilidad por parte del país de destino. Por ejemplo, un gobierno podría establecer una visa de entrada costosa, reducir la cuota de visas, poner una cuota de personas - que es un límite anual de cuántas personas de una nacionalidad en particular pueden llegar al país de destino - o requerir a cada individuo el tener una cantidad de dinero en efectivo al momento de cruzar la frontera. Además de esta pre-selección, aquí se plantea otro estrato adicional (el 6 en la Figura 1) para reflejar la burocracia post-migratoria, en la cual después de la migración, el país de destino continúa 
los procesos de control y de gobernabilidad, con el fin de intentar filtrar a los inmigrantes "deseables" de los "indeseables". El fin extremo de los indeseables resulta en la expulsión de los individuos; la deportación es constitutiva de "la criminalización de la inmigración" (Domenech, 2015, p. 170).

\section{La legalidad}

A pesar de haber experimentado un giro conceptual hacia el paradigma de movilidad humana, este término de legalidad no se aplica a todos los migrantes por igual y en todos los momentos de la trayectoria migratoria. Las clasificaciones legales de las personas a través de documentos migratorios resultan en la deseabilidad y la legalidad de solo algunos de los individuos (aquellos que cumplan las reglas de los estratos 4, 5 y 6). La "ilegalidad" como concepto es un producto de las leyes de inmigración de cada país: los inmigrantes sin documentos están criminalizados y definidos como ilegales por dichas leyes (De Genova, 2002, p. 439). A través de los pasos formales de los documentos migratorios, la burocracia requerida forma no solo una figura legal como inmigrante, sino que crea también la idea de una persona ilegal e irregular. Es aquí entonces - y nuevamente a través del rol del Estado migratorio - en donde se encuentra el origen de la criminalización en la migración (Bigo, 2004), también denominado como crimmigration por Stumpf (2006).

Entre los varios modelos de gobernabilidad existentes, el principal usado en Sudamérica está basado en el desarrollo humano para las migraciones (Mármora, 2010, p. 71). Esta aparente convergencia de modelo de gobernabilidad en el continente es, en parte, debido a que los Estados sudamericanos demuestran intereses traslapados y visiones similares - pero no necesariamente políticas públicas parecidas - con respecto a los derechos humanos (Finn et alii, 2019). Pero esta táctica de la gobernabilidad es solo una convergencia aparente: representa más bien una fachada retórica porque resulta en políticas públicas que poseen "(...) la misma finalidad que las políticas más abiertamente restrictivas" y por eso son "políticas de control con rostro humano" (Domenech, 2013, p. 2). En relación a la migración, los derechos humanos están en realidad yuxtapuestos a las restricciones de visas y tasas crecientes de detención y expulsión en Sudamérica (Domenech, 2013, p. 3; Domenech, Pereira, 2017, p. 99). La definición de legalidad es paralela al concepto de deportabilidad de las personas (De Genova, 2002). Una vez que los individuos entran en el país de destino, superando la frontera del estrato 5 de la Figura 1, existe la expulsión como una práctica política para eliminar los migrantes indeseables. Entonces, la expulsión es posible solo para aquellos presentes en los estratos 6 y 7 .

Las categorías legales de migrantes son una forma de control ex post de la soberanía estatal y política de un territorio. Sería difícil contradecir el 
derecho de cada Estado de poder ejercer dicho control en su territorio (pero para esta discusión normativa, véase Bauböck, 2012). El estrato 4 de la Figura 1 resulta en la construcción final del grupo acotado que será permitido para poder cruzar la frontera. Una vez este grupo supera la frontera (estrato 5), el estrato 6 de la burocracia post-migratoria es la única ruta legal a través de la cual los individuos pueden vivir y trabajar en el destino. A cambio de estas obligaciones y requisitos estatales, en muchos países el individuo puede luego acceder a los beneficios sociales, como por ejemplo, la seguridad de la policía, educación o atención médica. Se puede observar que algunos individuos no logran obtener una visa, ni superar la burocracia, pero igualmente pasan por la frontera de alguna manera, Ilegando al estrato 7. El "castigo" o penalización siguiendo el vocabulario de la criminalización - por haber saltado los estratos de la burocracia, se genera incentivos inversos: por ejemplo, una disminución al acceso y posibilidades de interactuar con dichos sistemas e instituciones formales en el país de destino. En suma, los procesos burocráticos de filtro de individuos (los estratos 4, 5 y 6 ) tienen consecuencias porque son factores claves de las condiciones ex ante y de los procesos subsiguientes de la participación de los inmigrantes.

\section{Conclusión}

En una época global compuesta de varias democracias con fronteras más libres, con una amplia aceptación de derechos humanos, con la existencia de organizaciones supra-nacionales y las conexiones virtuales y transnacionales entre individuos y mercados, se podría argumentar la gradual pero segura disminución del rol estatal, o la desterritorialización del Estado actual. Sin embargo y de acuerdo con lo que subrayan Hollifield y Wong (2015), el Estado continúa siendo un actor relevante en fenómenos de la migración internacional. Este estudio ha mostrado el rol insoslayable del Estado migratorio como un factor fundamental e influyente en los procesos migratorios, en cuanto se relaciona activamente con individuos, en definir la burocracia prey post-migratoria e influenciar indirectamente la estratificación en términos de participación formal. La contribución principal es definir los estratos, en manera sistemática, durante la trayectoria migratoria - especialmente la adición de la burocracia pre- y post-migratoria - a la literatura del control de fronteras y la legalidad.

Sin transmitir un sentido de omnipotencia, los Estado migratorios contemporáneos establecen el estatus legal que define quiénes disfrutan cuáles derechos, y quiénes no, en algún momento y contexto político dado. El rol estatal entonces consiste en definir el contexto legal y burocrático de los estratos dentro de la Figura 1 de la aproximación teórica-conceptual, ex ante y ex post de la migración. Los individuos toman decisiones bajo las estructuras, 
reglas, instituciones y fronteras de países. Regresando al título de este análisis, la relación entre la agencia del individuo y el Estado democrático no es una relación competitiva. Es decir, la evolución y los cambios en las instituciones que gobiernan los fenómenos sociales suceden debido a la agencia y acción de los individuos en la sociedad (Ostrom, 2011). Los individuos racionales ejercen su agencia pero confrontan las opciones limitadas y contextualizadas, dado los estratos burocráticos que emanan desde los países de destino. El Estado paradójicamente adquiere una única relevancia en la trayectoria migratoria desde el momento inicial en el que alguien comienza a considerar la posibilidad de migrar. Entonces los posibles inmigrantes no son solo auto-seleccionados, sino también originariamente acotados por el país de destino desde el principio, haciendo así que el fenómeno migratorio coincida conceptualmente entre la agencia del individuo y el Estado.

Los estratos detallados en la aproximación teórica-conceptual (Figura 1) alimentan un proceso político y legal de reducción en la posible población de migrantes que llegan a, y posteriormente participan en, un nuevo territorio. El grupo que llega a un país de destino ya posee una relación político-legal formal con el Estado de destino que afecta de forma única los procesos migratorios. Estas relaciones pre-existentes pueden servir como objetos de análisis más exhaustivos en futuras investigaciones sobre migración, incluso el cómo estas relaciones entre individuos personifican inherentemente los sistemas y procesos globales. En la aproximación, la población reducida sirve también como un punto de partida para futuros estudios acerca de la posibilidad de la integración y participación (política) formal de los inmigrantes en un país.

Finalmente, la aproximación teórica-conceptual refina las condiciones planteadas por Hollifield (2004, p. 885) que argumenta que las fuerzas económicas y sociológicas son condiciones necesarias para la ocurrencia de fenómenos de migración, pero las condiciones suficientes son aquellas legales y políticas. En la Figura 1, se observa que las condiciones necesarias ocurren en los primeros tres estratos. Los estratos estatales 4 y 6 de burocracia prey post-migración no son necesarios, pero su ausencia reduce drásticamente las oportunidades de participación formal que los individuos confrontarán en el país de destino. Cuando el 4 y 6 de la burocracia están combinados con el estrato 5 de la frontera, se convierten en un conjunto suficiente para generar la posibilidad de entrar en el estrato 7. De esta forma, se agrega el estrato 5 de la frontera como la única condición necesaria y suficiente para estar en el estrato 7 del grupo de inmigrantes. No obstante, para obtener la máxima posibilidad de participar desde el estrato 7, la combinación de 4, 5 y 6 es necesaria y suficiente. Para complementar la popularidad de estudiar el control de fronteras en particular, aquí se plantea que es la seguridad de la frontera junto con la burocracia pre- y post-migratoria que son relevantes en 
términos de las oportunidades de la participación de los inmigrantes en el país de destino.

\section{Agradecimientos}

Agradezco a Eva Hamamé, Pablo Paniagua y Sebastián Umpierrez de Reguero por ayudar a clarificar y mejorar el contenido de los argumentos. También agradezco a los organizadores y los participantes de la IV Escuela Internacional de Posgrado en septiembre 2017, especialmente a Cristián Doña-Reveco, Carolina Stefoni y Eduardo Domenech.

\section{Referencias}

ALVITES BAIADERA, Angélica. Peruanos en el cruce de frontera hacia Argentina. Reflexiones sobre las políticas de control migratorio desde las subjetividades de los migrantes. REMHU, Revista Interdisciplinar da Mobilidade Humana, v. 26, n. 53, p. 171-186, 2018.

ARRIGHI, Jean-Thomas; BAUBÖCK, Rainer. A Multilevel Puzzle: Migrants' Voting Rights in National and Local Elections. European Journal of Political Research, v. 56, n. 3, p. 619-639, 2017.

BAUBÖCK, Rainer. Transnational Citizenship: Membership and Rights in International Migration. Aldershot: Edward Elgar, 1994.

BAUBÖCK, Rainer. Migration and Citizenship: Normative Debates. In: ROSENBLUM, Marc R.; TICHENOR, Daniel J. (orgs.). Oxford Handbook of the Politics of International Migration. Oxford University Press, 2012.

BERRY, John W. Immigration, Acculturation, and Adaptation. Applied Psychology: An International Review, v. 46, n. 1, p. 5-68, 1997.

BIGO, Didier. Criminalisation of 'Migrants': The Side Effect of the Will to Control the Frontiers and the Sovereign Illusion. In: BOGUSZ, Barbara; CHOLEWINSKI, Ryszard; CYGAN, Adam; SZYSZCZAK, Erika (orgs.). Irregular Migration and Human Rights: Theoretical, European and International Perspectives. Leiden and Boston, MA: Marinus Nijhof Publishers, 2004.

BIGO, Didier. Vigilancia electrónica a gran escala y listas de alerta: ¿Productos de una política paranoica? REMHU, Revista Interdisciplinar da Mobilidade Humana, v. 23, n. 45, p. 11-42, 2015.

DE GENOVA, Nicholas P. Migrant "Illegality" and Deportability in Everyday Life. Annual Review of Anthropology, v. 31, p. 419-447, 2002.

DE GENOVA, Nicholas P.; MEZZADRA, Sandro; PICKLES, John (orgs.). New Keywords: Migration and Borders. Cultural Studies, v. 29, n. 1, p. 55-87, 2015.

DOMENECH, Eduardo. "Las migraciones son como el agua": Hacia la instauración de políticas de "control con rostro humano". Polis, v. 35, p. 2-17, 2013.

DOMENECH, Eduardo. Inmigración, anarquismo y deportación: La criminalización de los extranjeros "indeseables" en tiempos de las "grandes migraciones". 
REMHU, Revista Interdisciplinar da Mobilidade Humana, v. 23, n. 45, p. 169196, 2015.

DOMENECH, Eduardo; PEREIRA, Andrés. Estudios migratorios e investigación académica sobre las políticas de migraciones internacionales en Argentina. Íconos. Revista de Ciencias Sociales, v. 58, p. 83-108, 2017.

ESTUPIÑÁN SERRANO, Mary Luz. Acerca de la gobernanza y la gestión migratoria. Crítica del papel de la OIM en el gobierno de la migración internacional y regional. Documentos de trabajo, CLACSO, n. 33, 2013.

FAIST, Thomas. Transnationalization in International Migration: Implications for the Study of Citizenship and Culture. Ethnic and Racial Studies, v. 23, n. 2, p. 189222, 2000.

FINN, Victoria; DOÑA-REVECO, Cristián; FEDDERSEN, Mayra. Migration Governance in South America: Regional Approaches versus National Laws. In: GEDDES, Andrew; ESPINOZA, Marcia V.; HADJ ABDOU, Leila; BRUMAT, Leiza (orgs.). The Dynamics of Regional Migration Governance. Edward Elgar Publishing, 2019.

FREEMAN, Gary P. Modes of Immigration Policies in Liberal Democratic States. International Migration Review, v. 29, n. 4, p. 881-902, 1995.

GEIGER, Martin; PÉCOUD, Antoine (orgs.). The Politics of International Migration Management: Migration, Minorities and Citizenship. London: Palgrave Macmillan, 2010.

GLICK SCHILLER, Nina. A Global Perspective on Transnational Migration: Theorising Migration without Methodological Nationalism. In: BAUBÖCK, Rainer; FAIST, Thomas (orgs.). Diaspora and Transnationalism: Concepts, Theories and Methods. Amsterdam University Press, 2010a.

GLICK SCHILLER, Nina. A Global Perspective on Migration and Development. In: GLICK SCHILLER, Nina; FAIST, Thomas (orgs.). Migration, Development, and Transnationalization: A Critical Stance. Berghahn Books, $2010 b$.

HAMPSHIRE, James. The Politics of Immigration: Contradictions of the Liberal State. Cambridge: Polity Press, 2013.

HOLLIFIELD, James F. Immigration Policy in France and Germany: Outputs versus Outcomes. The Annals of the American Academy of Political and Social Science, v. 485, p. 113-128, 1986.

HOLLIFIELD, James F. The Emerging Migration State. International Migration Review, v. 38, n. 3, p. 885-912, 2004.

HOLLIFIELD, James F.; MARTIN, Philip L.; ORRENIUIS, Pia M. (orgs.). Controlling Immigration: A Global Perspective. Stanford University Press, 2014.

HOLLIFIELD, James F.; WONG, Tom K. The Politics of International Migration: How Can We 'Bring the State Back In'? In: BRETTELL, Caroline B.; HOLLIFIELD, James F. (orgs.). Migration Theory: Talking Across Disciplines. Routledge, 2015.

KALM, Sara. Governing Global Migration (Tesis de Doctorado). Lund University, Statsvetenskapliga institutionen, Lund, 2008. 
MÁRMORA, Lelio. Modelos de Gobernabilidad Migratoria. La Perspectiva Política en América del Sur. REMHU, Revista Interdisciplinar da Mobilidade Humana, v. 35, p. 71-92, 2010.

MASSEY, Douglas S.; ARANGO, Joaquin; HUGO, Graeme; KOUAOUCl, Ali; PELLEGRINO, Adela; TAYLOR, J. Edward. Theories of International Migration: A Review and Appraisal. Population and Development Review, v. 19, n. 3, p. 431-466, 1993.

MEZZADRA, Sandro. Multiplicação das fronteiras e práticas de mobilidade. REMHU, Revista Interdisciplinar da Mobilidade Humana, v. 23, n. 44, p. 11-30, 2015.

OSTROM, Elinor. Background on the Institutional Analysis and Development Framework. The Policy Studies Journal, v. 39, n. 1, p. 7-27, 2011.

POWELL Jr., G. Bingham. Political Representation in Comparative Politics. Annual Review of Political Science, v. 7, p. 273-296, 2004.

RIVERA SÁNCHEZ, Liliana. Vínculos y prácticas de interconexión en un circuito migratorio entre México y Nueva York. Buenos Aires: CLACSO, 2012.

STUMPF, Juliet. The Crimmigration Crisis: Immigrants, Crime, and Sovereign Power. American University Law Review, v. 56, n. 2, p. 367-419, 2006.

VIVEROS, Mara. La interseccionalidad. Una aproximación situada a la dominación. Debate feminista, v. 52, p. 1-17, 2016.

WALDINGER, Roger D.; FITZGERALD, David. Transnationalism in Question. American Journal of Sociology, v. 109, n. 5, p. 1177-1195, 2004.

WIMMER, Andreas; GLICK SCHILLER, Nina. Methodological Nationalism and Beyond: Nation-State Building, Migration and the Social Sciences. Global Networks, v. 2, n. 4, p. 301-334, 2002. 